\title{
Revista Internacional de Filosofia
}

Volumen IX (2004) • ISSN: $1136-4076$

\section{SUMARIO}

ESTUDIOS

Luis Alvarez Colin

Mauricio Beuchot

José Calvo Gonzalez

Sixto J. Castro

La hermenéutica analogica: aportación fundamental de la filosofia mexicana

Los pitagóricos y la analogía. La visión de Maria Zambrano 27-40

Jan Patocka y la Carta 77. Antropología fenomenológica crítica y

activismo de los derechos humanos

$41-58$

Una teoria moral del arte. Moralismo moderado, cpistémico y

sistémico

59-76

Juan A. Garcia Gonzailez

H. C. Felipe Mansilla

Teorias y actitudes escépticas en la Antiguedad

77.94

Apuntes eriticos sobre el postmodernismo y teorias afines 95-106

Cristina Märquez Rodilla

En tomo a los avatares del placer virtual

$107-122$

Pascual F. Martinez Freire

Psicología y materialismo

$123-142$

José Rubio Carracedo

Por una ćtica transcultural

$143-160$

NOTAS CRITICAS

Antonio Gallardo Cervantes

Lévinas frente a la modernidad

161-174

Luis Puelles Romero

En torno a la existencia de una estética nietzscheana

175-184

TRADUCCIÓN CRITICA

Maurice Merleau-Ponty

Prólogo a la Fenomenología de la percepción

(Prescntación, traducción y apéndice de Benito Arias Garela)

INFORME BIBLIOGRÁFCO

Ángel Ramirez Medina Bibliografía sobre Albert Camus

213-236

RESEÑAS

237.250

LIBROS RECIBIDOS

FONDO EDTTORIAL. Contro.stes

253-270 


\title{
Psicología y materialismo
}

\author{
PASCUAL F. MARTÍNEZ-FRFIRE \\ Universidad de Málaga
}

\section{RESUMEN}

Iniéalmente se distingue entre materialismo ontológico y materialismo mental. Se analizan las principales tesis de los tres tipos de materialismo mental (clásico o teoria de la identidad, eliminativo y de sobreveniencia), presentando objeciones a todos ellos. Se concluye defendiendo el funcionalemergentismo, que combina la tesis funcionalista en ciencias cognitivas y la tesis emergentista en psicología e informática.

PALABRAS CLAVE

\section{FUNCIONALEMERGENTISMO, MATERIALISMO MENTAL, PSICOLOGIA VERSUS} NEUROCIENCIA, SOBREVENIENCLA.

\section{ABSTRACT}

Firstly a distietion is made between ontokogical materialism and mental materialism. Then the main theses concerning the three kinds of mental materialism (classic or identity-theory, eliminative and supervenience materialism) are analyzed, raising objections to them. The concluding claim is functionalemergentism, combining the functionalist thesis in cognitive science and the emergentist thesis in psychology and computer science.

KEYWORDS

\section{FUCTIONALEMERGENTISM, MENTAL MATERIALISM, PSYCHOLOGY VERSUS} NEUROSCIENCE, SUPERVENIENCE

\section{DOS TIPOS (PARA EMPEZAR) DE MATERIALISMO}

EN TÉRMINOS COLOQUIALES, QUE TODAVIA NO SE REHEREN a la distinción entre dos tipos de materialismo que deseo trazar, podemos seĩalar dos contextos distintos, uno negativo y otro positivo, para el término «materialista». En efecto, es frecuente escuchar la frase siguiente: «no piensas más que en comer, beber y en el sexo, eres un materialistas; en este contexto coloquial, el término

Q Contrastes. Revista Internacional de Filosofia, vol, IX (2004), pp. 123-142. ISSN: 1136-4076 Sección de Filosofía, Universidad de Malaga, Facultad de Filosoffa y Letras Campus de Teatinos, E-29071 Málağa (España) 
«materialista» tiene una carga semántica negativa, $\mathrm{y}$ la persona aludida, a la que atribuimos una exclusiva preocupación por sus propios placeres, es valorada negativamente. En cambio, también puede escucharse la frase siguiente: «Fulano, como materialista, no cree ni en los fantasmas ni en los espíritus, sino en los hechos científicos»; ahora, en este otro contexto coloquial, el término «materialista» tiene una carga semántica positiva, y la persona aludida es valorada de forma positiva.

Este segundo contexto, el del materialismo unido a la ciencia, tiene que ver con el materialismo ontológico, que pretendo distinguir del materialismo en filosofía de la mente (aunque también relacionarlos).

En general, y tal como he señalado en mi trabajo «A proximaciones científicas al problema de la conciencia» (2000) ${ }^{1}$, para el materialismo (ontológico) cualquier realidad es de índole material, con lo que cualquier entidad no-material es declarada no-existente, es decir, «materia» y «existencia» son términos lógicamente coextensivos (se aplican a las mismas entidades), de modo que todo lo que existe es material y todo lo material existe. Pero además las realidades materiales, esto es, las realidades sin más, son descritas utilizando categorías físicas y objetivas.

Pero entonces debemos intentar averiguar qué es la materia para la física. Werner Heisenberg (1901-1976), en su libro Das Naturbild der heutigen Physik (1955), recoge varios textos de autores (D'Alembert, La Mettrie y Ostwald) que conformaron la imagen del universo mecanicista y materialista. De entre ellos, me interesa, para nuestro propósito, el texto del químico alemán Friedrich Wilhelm Ostwald (1853-1932). Este científico, en sus Vorlesungen über Naturphilosophie (de 1902), señala la siguiente caracterización de la materia en la física y química del siglo XIX ${ }^{2}$. Toda materia se da en determinada cantidad; a la cantidad de materia se le llama generalmente masa. A su vez, la materia presenta ciertas diferencias cualitativas, que pueden reducirse a la existencia de 70 u 80 elementos (hoy hablaríamos de unos cien) no transformables unos en otros. Además, la materia posee extensión espacial y delimitación en la forma. A la materia se le atribuye también impenetrabilidad, en el sentido de que dos distintos pedazos de materia no pueden estar a un mismo tiempo en un mismo lugar. Finalmente la materia se tiene por indestructible. Ostwald añade, en este texto, que a veces se distingue entre esas propiedades enumeradas de la materia, que se consideran esenciales, $y$ otras propiedades generales que, a pesar de hallarse en toda materia, no forman parte esencial de su concepto. Entre ellas se cuenta la inercia, o sea la capacidad de permanecer en determi-

1 Pp. $185-186$.

2 Werner Heisenberg, La imagen de la naturaleza en la física actual, pp. 115-116. 
nado estado de movimiento, la gravedad, la divisibilidad y la porosidad. Pero nuestro autor precisa que no hay unanimidad respecto de las propiedades que son esenciales, ni respecto de las propiedades generales e incluso a menudo se abandona la distinción entre unas y otras.

Por su parte Ostwald propone sustituir esa concepción de la materia por el concepto de energía ${ }^{3}$, ya que sólo la energía se manifiesta sin excepción en todos los fenómenos naturales conocidos. Más aún (y esto nos interesa en especial) propone concebir a la conciencia como cierta propiedad de una forma peculiar de energía nerviosa, a saber, la energía que se ejecuta en el órgano central (cerebro), con lo que también los procesos internos de la conciencia son de carácter energético. En suma, para Ostwald las distintas realidades son distintas formas de energía.

El propio Heisenberg, en la obra que nos ocupa ${ }^{4}$, extrae algunas conclusiones de su recorrido por los textos que presenta. Por un lado, señala que la ciencia moderna en sus comienzos se distingue por una deliberada modestia, formulando enunciados válidos para dominios estrictamente delimitados. En segundo lugar, señala que en el siglo XIX aquella modestia se pierde en gran parte, la Física aspira a ser una Filosofía y muchas veces se proclama que toda verdadera filosofía ha de ser únicamente ciencia de la naturaleza ${ }^{5}$. En tercer lugar, Heisenberg sostiene que la Física en su tiempo (1955) está volviendo a su primitivo comedimiento. Y finalmente el físico alemán propugna que la Física deje en suspenso la decisión sobre qué sean los cuerpos, la materia, la energía, etc., para alcanzar conocimientos sobre propiedades singulares de los fenómenos designados con esos términos, conocimientos que luego pueden conducir a auténticas concepciones filosóficas. En suma, para Heisenberg la caracterización de la materia es una cuestión filosófica, no una cuestión física.

Mario Bunge, filósofo argentino-canadiense conocido por su defensa del materialismo ontológico, asocia, como vimos en el segundo ejemplo coloquial, materialismo y ciencia. En su libro titulado precisamente Materialismo y Ciencia (1981) intenta establecer el concepto contemporáneo de materia, declarando que un objeto material es «un objeto que puede estar por lo menos en dos estados, de modo que puede saltar de uno a otro" ${ }^{6}$, y también que «todo ente material es cambiable cuando menos en lo que se refiere a su posición respecto de otros entes materiales»? Según ello la materia queda definida por la mutabilidad.

3 O.c., pp. $118-120$.

4 O. c., pp. 148-149.

5 Obsérvese la anticipación de la naturalización de la filosofía, que nuestro científico rechaza.

6 Mario Bunge, Materialismo y Ciencia, p. 35 ,

7 O.c., p. 29.

Contrastes vol. IX (2004) 
Personalmente no creo que ésta sea una caracterización adecuada o suficiente. No es por molestar (aunque Bunge se molestaría) pero el paso del alma del purgatorio al cielo es un cambio, pero ello no convierte al alma en un ente material. Pero en realidad, la caracterización de la materia por Bunge es circular, ya que caracteriza la materia como lo que estudia la ciencia ${ }^{8}$, y caracteriza la ciencia como el estudio de la materia9.

En mi opinión, el materialismo ontológico es una creencia filosófica y, a su vez, la materia sólo puede caracterizarse en términos de sentido común o, si se prefiere, física popular. En efecto, consideramos realidades materiales aquéllas que pueden ser objeto de nuestros cinco sentidos, que podemos ver, oír, gustar, oler o tocar, y también podemos considerar realidades materiales aquéllas que inferimos a partir de las realidades sensoriales y que conjeturamos que también son materiales como éstas. Por ejemplo, esta página escrita es una realidad material, ya que puedo verla, olerla o tocarla (no parece que pueda oírla ni es recomendable que la guste), pero también consideramos una realidad material el árbol del cual inferimos que procede esta hoja de papel, aunque tal árbol no sea objeto de sensación (pero podría serlo). En cambio, el monstruo del Lago Ness no es una realidad material, ya que nadie (de manera fehaciente) lo ha visto, oído o tocado. Pues bien, el materialismo ontológico sostiene que sólo existen realidades materiales, esto es, realidades que pueden ser objeto de nuestros sentidos o bien que pueden ser inferidas a partir de los objetos sensibles y presumir que también son sensibles.

Del materialismo ontológico se distingue (aunque cabe relacionarlos) el materialismo en filosofía de la mente. Éste sostiene que los procesos mentales se identifican o reducen, en mayor o menor medida, con los procesos cerebrales, de tal manera que la psicología como ciencia se elimina o se reduce en gran medida a la neurociencia. Según los grados de esta identificación o reducción tendremos los distintos tipos de este materialismo, que veremos más adelante.

Para entender bien el materialismo en filosofía de la mente, que podemos denominar materialismo mental o materialismo de la mente, es preciso introducir varias aclaraciones.

En primer lugar, mientras que el materialismo ontológico es una tesis que afirma que sólo hay realidades materiales (quedando claro su carácter ontológico), en cambio el materialismo mental es una tesis de filosofía de la ciencia que elimina en mayor o menor medida la psicología en favor de la neurociencia.

8 «Después de todo la materia es lo que estudia la ciencia» (o. c., p. 28).

9 «La ciencia contemporánea puede caracterizarse como el estudio de objetos materiales por medio del método científico y con el fin de encontrar y sistematizar las leyes de tales objetos» (o. c., p. 29). 
Debe quedar claro que el materialista mental puede admitir algunas realidades no-materiales, como por ejemplo un Dios espiritual, mientras que el materialista ontológico, aunque tiene que considerar materiales los procesos mentales, puede mantener la independencia de la psicología respecto de la neurociencia. En cambio, el materialista mental es forzosamente un materialista ontológico cuando se trata de los procesos mentales ( $\mathrm{y}$ ésta es la relación anunciada entre ambos materialismos).

En segundo lugar, también debe aclararse qué entendemos por procesos mentales ${ }^{10}$. Los procesos mentales son una colección de conjuntos de procesos entre los cuales encontramos sensaciones, percepciones, creencias, inferencias, recuerdos, imágenes, voliciones y emociones. Ello quiere decir que adoptamos una global teoría causal de la mente ${ }^{11}$, contraria al conductismo, según la cual los procesos mentales son algo interno y distinto de la conducta y asimismo factores causales de la conducta (aunque a veces los procesos mentales no producen conducta).

En tercer lugar, la aparición del materialismo mental, a partir de 1956 (tal como veremos) supone el impacto tardío de la neurociencia en la filosofía de la mente. Como es sabido, la neurociencia surgió a fines del siglo XIX con Santiago Ramón y Cajal (1852-1934) cuando estableció que el tejido nervioso no es una masa continua sino una red de células delimitadas, las neuronas; en 1904 (hace ahora cien años) Cajal publicó su Textura del sistema nervioso del hombre y de los vertebrados, que constituye la obra fundacional de la neurociencia. En 1906 el inglés Charles Sherrington (1857-1952) estudia en detalle las sinapsis o contactos entre neuronas en The Integrative Action of the Nervous System. Posteriormente, en los años veinte el inglés Edgar Adrian (1889-1977) realizó registros eléctricos en todo el sistema nervioso, y el americano Wilder Penfield (1891-1976) creó en 1934 el Instituto Neurológico de Montreal (Canadá). Desde entonces la neurociencia ha avanzado constantemente, aunque la Society for Neuroscience sólo se fundó en 1970, hubo que esperar a la década de 1990 para que se produjeran los más notables avances, y aún hoy no constituye una ciencia completa con una doctrina común aceptada en todos sus aspectos.

\section{El MATERIALISMO MENTAL CLÁSICO: LA TEORÍA DE LA IDENTIDAD MENTE-CEREBRO}

Existe consenso en considerar el artículo del filósofo australiano Ullin T. Place (1925-2000) titulado «Is Consciousness a Brain Process?» (1956), donde Sherrington es citado, el inicio del materialismo clásico en filosofía de

10 Véase mi trabajo *Mente, inteligencia y espíritu» (2000), pp. 693-696.

11 Véase mi libro La nueva filosofía de la mente (2002), pp. 77-78. 
la mente. Place habla de conciencia, en vez de procesos mentales en general, como si fueran expresiones sinónimas, pero esta indefinición entre procesos conscientes en particular y procesos mentales en general es muy corriente entre los primeros filósofos de la mente e incluso entre los primeros psicólogos, aunque la teoría del inconsciente de Sigmund Freud (1856-1939) ya estaba al alcance de cualquiera a principios del siglo XX. Para Place, podemos identificar la conciencia con un patrón de actividad cerebral, aunque admite que las operaciones requeridas para verificar enunciados acerca de la conciencia y enunciados acerca de procesos cerebrales son fundamentalmente diferentes. En suma, se abre camino en este trabajo la teoría de la identidad mente-cerebro, según la cual los procesos mentales se identifican con los procesos cerebrales, aunque pueden subsistir las explicaciones psicológicas (pero, podemos añadir, como explicaciones provisionales).

Place, en su trabajo póstumo «A Pilgrim's Progress? From Mystical Experience to Biological Consciousness» (2002), atribuye ${ }^{12}$ la idea de que la relación entre conciencia y actividad cerebral es una relación de identidad al psicólogo y conocido historiador de la psicología Edwin Boring (1886-1968). En efecto, este autor, en su libro The Physical Dimensions of Consciousness $(1933)^{13}$ sostiene que las entidades psicológicas son tan palpables como las físicas $^{14}$, añadiendo que en cualquier caso los datos de la introspección y los datos de la física están coordinados y en armonía, y concluye que el propósito de su libro es ponerlos juntos en un sistema cerrado.

Además de Place, el otro iniciador del materialismo mental clásico es el filósofo inglés-australiano John J. C. Smart. En su artículo «Sensations and Brain Processes» (1959), declara seguir los argumentos de Place en su trabajo de 1956 y, aunque habla de sensaciones, debe entenderse que al igual que hacía Wittgenstein (al que se refiere de modo explícito) cabe generalizar desde las sensaciones a la totalidad de los procesos mentales. Para Smart las sensaciones resultan ser procesos cerebrales, aunque los enunciados de sensaciones no puedan traducirse en enunciados de procesos cerebrales. Así pues, al igual que Place, Smart considera que los procesos mentales se identifican con los procesos cerebrales, pero queda espacio para la psicología. Nuestro autor concluye su trabajo diciendo que, si se acuerda que no hay ningún argumento filosófico convincente a favor del dualismo (entre mente y cerebro), y si el dualismo y la teoría del proceso cerebral son igualmente consistentes con los hechos, entonces los principios de economía y simplicidad parecen decidir a favor de la teoría del

12 *A Pilgrim's Progress» (2002), p. 36.

13 The Physical Dimensions of Consciousness (1933), p. 13.

14 No me parece que una idea $\mathrm{o}$ un sentimiento puedan considerarse pal pables. 
proceso cerebral. Evidentemente, cabe añadir, es más simple tener sólo procesos físicos que tener procesos físicos y además procesos mentales; la cuestión, me parece, es que además de simple puede ser también simplista.

En todo caso los autores más representativos del materialismo mental clásico (teoría de la identidad mente-cerebro) son los filósofos también australianos David M. Armstrong y David Lewis (1941-2001).

La teoría de la identidad mente-cerebro de Armstrong aparece ya expuesta y justificada en su artículo «The Nature of Mind» (1966), analizada en su libro A Materialist Theory of the Mind (1968) y reiterada en su reciente libro The Mind-Body Problem. An Opinionated Introduction (1999). Para Armstrong ${ }^{15}$, en el artículo de 1966, la pregunta por la naturaleza de la mente debe ir unida a la pregunta por la naturaleza del ser humano, ya que son los seres humanos los que tienen mente, es decir, tienen sensaciones, perciben, tienen creencias, emociones, propósitos o deseos. Ahora bien, en cuanto a la naturaleza del ser humano debemos atender a las respuestas de la ciencia actual, para la cual podemos dar una explicación completa del ser humano en términos puramente físico-químicos. Por tanto debemos intentar desarrollar una explicación de la naturaleza de la mente que sea compatible con la tesis científica de que los seres humanos no son sino mecanismos físico-químicos. Por otra parte, Armstrong, frente al conductismo, sostiene que los procesos mentales no pueden identificarse con la conducta, sino que son la causa interna de la conducta. Y nuestro autor concluye que la única causa de la conducta, en los seres humanos y en los animales superiores, es el funcionamiento físico-químico del sistema nervioso central; por tanto, podemos identificar los estados mentales con estados puramente físicos del sistema nervioso central.

A su vez, en A Materialist Theory of the Mind (1968), Armstrong analiza y desarrolla las tesis anteriores. En esta obra distingue de modo claro y explícito las dos etapas que llevan a la teoría materialista de la mente; en una primera etapa, se cumple el análisis causal de los conceptos mentales, es decir, la tesis de que los estados mentales son causa interna de la conducta, mientras que en la segunda etapa se identifican tales estados internos con los estados físicoquímicos del cerebro, proporcionando así el materialismo de estado central (reducción de la mente a los estados físicos del sistema nervioso central). Según esto, podemos añadir, la neurociencia es en última instancia la disciplina científica que explica los fenómenos psicológicos; pero la psicología no desaparece en este materialismo mental clásico y, tal como dice David Armstrong ${ }^{16}$, en

15 Véase mi exposición, más completa en algunos puntos, en La nueva filosofía de la mente (2002), pp. 78-81.

16 A Materialist Theory of the Mind (1968), pp. 78-79. 
«una primera aproximación» la mente o procesos mentales particulares son los efectos de estímulos y las causas de respuestas en el ser humano.

Finalmente, el libro de David Armstrong The Mind-Body Problem. An Opinionated Introduction (1999) constituye un libro de texto sobre teorías de la mente que no añade gran cosa a sus trabajos anteriores, sal vo precisar que tanto él como Lewis defienden lo que propiamente debe denominarse «teoría causal». En esta línea de argumentación, los estados mentales son estados aptos para producir o causar ciertos tipos (ranges) de conducta y, asimismo, son estados aptos para ser producidos o causados por ciertos tipos (ranges) de causas.

El otro gran representante del materialismo mental clásico es David Lewis. Sus trabajos centrales al respecto son los artículos «An Argument for the Identity Theory» (1966) y «Psychophysical and Theoretical Identifications» (1972). Yendo a lo esencial ${ }^{17}$, Lewis considera que la identificación entre los procesos mentales y los procesos cerebrales (identificación psicofísica) no consiste en postular la identificación entre entidades de una teoría (la psicología) y entidades de otra teoría (la neurociencia), sino que defiende que tal identificación puede ser resultado de una implicación (es decir, no ser objeto de postulación sino de conclusión lógica). Podemos presentar el argumento de Lewis en los siguientes términos: 1) los estados mentales se identifican con los causantes de conducta, 2) los estados neuronales se identifican con los causantes de conducta, y 3) luego los estados mentales se identifican con los estados neuronales. Debe aclararse que la primera premisa está apoyada por la ya citada teoría causal de la mente, mientras que la segunda premisa está apoyada en los descubrimientos de la neurociencia. En todo caso, la posición de Lewis tampoco elimina la psicología, ya que ésta tiene como tarea estudiar (aunque en primera instancia) las causas de la conducta, pero la última palabra explicativa la tiene la neurociencia.

\section{Algunas observaciones CRíticas al materialismo Clásico}

Mi propósito principal al escribir este artículo es aclarar las tesis de los distintos materialismos en filosofía de la mente. Por ello, y también por limitaciones de espacio, me ceñiré, en cada caso, a las observaciones críticas que me parecen fundamentales.

Mi objeción básica y general al materialismo en filosofía de la mente (es decir, a la tesis que establece que los procesos mentales se reducen o identifican en mayor o menor medida con los procesos cerebrales) se encierra en lo que he denominado el argumento antimaterialista de las máquinas ${ }^{18}$. La ciencia

17 Para una exposición más detallada, véase mi libro La nueva filosofía de la mente (2002), pp. 82-87.

18 O. c., pp. $86-87$. 
de la inteligencia artificial, una de las ciencias cognitivas básicas junto con la psicología, ha llegado a construir computadores «inteligentes», es decir, máquinas que realizan tareas que en los humanos atribuimos a su inteligencia (como demostrar teoremas, ganar una partida de ajedrez o establecer un diagnóstico médico). Parece, por tanto, normal que hablemos de mentes mecánicas, esto es, que hablemos de que los computadores tienen procesos mentales, ya que tienen sensaciones (entradas de información), recuerdos (memorias de varios tipos) e incluso frecuentemente realizan inferencias, con lo que entonces exhiben inteligencia, puesto que la inteligencia va unida a la capacidad de razonar o inferir. Sin embargo, en estas máquinas no existen procesos neurológicos, por la sencilla razón de que no se componen de neuronas o células nerviosas. En consecuencia, la identidad (en mayor o menor medida) entre procesos mentales y procesos neurológicos queda refutada, ya que se dan procesos mentales enteramente ajenos a los procesos neurológicos. En este punto, resulta interesante poner de relieve que el desarrollo de las máquinas, tarea alentada tradicionalmente por el materialismo mecanicista, se vuelve contra tal materialismo en su tentativa de reducir la mente (en mayor o menor medida) a los mecanismos físico-químicos del sistema nervioso central.

En cuanto a la teoría de la identidad mente-cerebro de Armstrong, éste señala en A Materialist Theory of the Mind ${ }^{19}$ que la identificación de los estados mentales con los estados físico-químicos del cerebro es una apuesta (bet), una apuesta casi tan buena, en el actual estado del conocimiento, como la identificación del gen con la molécula de ADN. Sin embargo, David Armstrong pierde de vista que ambas situaciones no son comparables. El gen era, antes de su identificación con el ADN, la unidad de herencia biológica concebida en términos abstractos e indeterminados, mientras que un proceso mental particular (como un sentimiento de odio o una percepción visual) es algo concreto y determinado, empíricamente contrastable.

En el caso de la teoría de la identidad mente-cerebro de David Lewis, la identificación psicofísica aparece ya no como una propuesta, sino como una conclusión lógica implicada por premisas supuestamente científicas. Sin embargo, entiendo que ambas premisas pueden ser puestas en duda. En primer lugar, la identificación entre estados mentales y factores causales de la conducta no es en general verdadera, ya que existen estados mentales que no producen efecto alguno en la conducta (como, por ejemplo, sentimientos que no se manifiestan en comportamiento alguno o imágenes mentales que permanecen privadas). $Y$ en segundo lugar, la identificación entre estados neuronales y factores causales de la conducta tampoco es en general verdadera, ya que existen procesos neu-

19 A Materialist Theory of the Mind (1968), pp. 89-90.

Contrastes vol. IX (2004) 
rológicos que no producen acciones que puedan calificarse de conducta en el sentido que interesa al psicólogo (como, por ejemplo, los procesos de la zona de la corteza cerebral motora responsable de los movimientos de la lengua cuando ésta interviene en la masticación de alimentos).

\section{EL MATERIALISMO MENTAL ELIMINATIVO: PRESENTACIÓN Y OBSERVACIONES CRITICAS}

El materialismo mental clásico que acabamos de ver deja cierto espacio a la psicología, ya que las observaciones psicológicas constituyen un conjunto de elementos primarios y las explicaciones psicológicas pueden entenderse como explicaciones preliminares, todo ello previo a las auténticas y definitivas explicaciones que nos dará la neurociencia. Sin embargo, cabe un tratamiento menos condescendiente con la psicología, consistente en eliminar sin más esta ciencia en favor de la neurociencia. Tal es la tesis del materialismo eliminativo, propia de algunos filósofos de la ciencia aunque (claro está) no propia de los psicólogos.

El filósofo austriaco-americano Paul Feyerabend (1924-1994) fue, al parecer, el primero en defender este punto de vista. En efecto, en su artículo «Materialism and the Mind-Body Problem» (1963), después de discutir los argumentos principales, según él, en contra del materialismo, concluye que no existe una sola razón por la cual deba abandonarse la tentativa de proporcionar una explicación exclusivamente fisiológica de los seres humanos, o por la cual los fisiólogos deban dejar el alma (soul) fuera de sus consideraciones.

Sin embargo, el principal promotor del materialismo mental eliminativo es el filósofo canadiense-americano Paul Churchland. Sus obras centrales al respecto son el artículo «Eliminative Materialism and the Propositional Attitudes» (1981) y el libro Matter and Consciousness (1984). En realidad el ataque de Churchland es doble, ya que su objetivo inmediato es el rechazo de la psicología popular pero, al mismo tiempo, su objetivo mediato es la psicología científica, que considera inevitablemente impregnada de psicología popular. El psicólogo y filósofo americano Jerry Fodor, en varios de sus escritos, en particular en Psychosemantics (1987), defiende la psicología popular (folk psychology) o psicología de sentido común, es decir, los conocimientos psicológicos que todos (hayamos estudiado o no psicología) poseemos de hecho acerca de los procesos mentales y de su relación con la conducta; además, para Fodor, la psicología científica debe incorporar buena parte de las intuiciones de la psicología popular. Pues bien, Paul Churchland considera que la psicología popular es una teoría falsa y que la psicología científica debe ser eliminada en favor de la neurociencia. 
Para él ${ }^{20}$ y en primer lugar, la psicología popular fracasa de modo amplio en la explicación y predicción de los procesos mentales (no sabemos lo que es el sueño, no comprendemos cómo se desarrolla el aprendizaje, no sabemos cómo funciona la memoria, no conocemos qué es la enfermedad mental) de tal manera que las cosas más centrales acerca de nosotros permanecen casi enteramente misteriosas dentro de la psicología popular. En segundo lugar, si la psicología popular ha sobrevivido hasta ahora no es a causa de su carácter correcto, sino porque los fenómenos mentales son tan difíciles de explicar que cualquier recurso explicativo, aunque sea débil, consigue mantenerse. $Y$ en tercer lugar, una neurociencia que no refleje la psicología popular tendrá mucho más éxito explicativo que una neurociencia que refleje la estructura psicológica de nuestro sentido común. Nuestro filósofo defiende la idea ${ }^{21}$ de que cuando la neurociencia haya madurado y se haya establecido la superioridad de su nueva estructura, entonces seremos capaces de comenzar a concebir de otra manera nuestros estados y actividades internas. Entonces nuestras explicaciones de la conducta recurrirán a cosas tales como nuestros estados neurofarmacológicos, la actividad neuronal en áreas anatómicas especializadas y cualesquiera otros estados que parezcan relevantes para la nueva teoría. Y entonces también nuestra introspección privada será transformada y podrá ser profundamente aumentada gracias a la estructura más precisa y penetrante con la que será tratada. Todo ello supondrá una revolución conceptual de enorme magnitud así como grandes beneficios para la humanidad. En conclusión, nos propone Churchland, eliminemos la psicología popular, con ella la psicología científica, y apostemos por la neurociencia.

Personalmente creo que Paul Churchland argumenta dialécticamente al enfrentarse a la psicología popular ${ }^{22}$. La psicología del sentido común no es propiamente una teoría, ya que sus generalizaciones (por ejemplo, "una persona hará algo que desea") no son leyes estrictas, sino máximas o lugares comunes, que constituyen mecanismos útiles de explicación y predicción en casos sencillos y cotidianos. Nadie razonable pretende explicar exclusivamente con sus dotes naturales comportamientos complejos o estados de ánimo de personas extraordinarias (para eso están los psicólogos científicos). Y no siendo una teoría, tampoco es una teoría falsa, ni tiene sentido imputarle fracasos en tareas que no emprende.

Por otra parte, el lenguaje mentalista propio de la psicología popular (conceptos tales como deseo, creencia, temor, intención, pero también sentimiento,

20 Matter and Consciousness (1988), pp. 45-47.

21 O.c., pp. 4445.

22 Véase mi La nueva filosofía de la mente (2002), p. 71.

Contrastes vol. IX (2004) 
motivación o plan) no puede eliminarse de la psicología científica. Ahora bien, estos conceptos no aparecen en la neurociencia, razón que apunta a la imposibilidad de eliminar la psicología en favor de la neurociencia.

Finalmente hay una contradicción entre la aceptación por Churchland de la posibilidad de mentes mecánicas y la eliminación de la psicología en favor de la neurociencia (lo cual implica que los fenómenos mentales no son más que procesos neuronales). En efecto, en su trabajo en colaboración con su esposa Patricia Churchland «Could a Machine Think?» (1990), sostiene la tesis general de que la inteligencia artificial clásica tiene dificultades para crear máquinas que piensen, pero que en cambio la inteligencia artificial conexionista puede crear máquinas que piensen ${ }^{23}$. Recordemos que la inteligencia artificial clásica o simbólica, fundada por Allen Newell (1927-1992), Herbert Simon (19162001), John McCarthy y Marvin Minsky, en 1956, constituye una manera de hacer inteligencia artificial que, desde 1986, se ha visto complementada por la inteligencia artificial conexionista o subsimbólica de David Rumelhart y James McClelland. En términos generales, mientras que la primera es digital, ya que opera con pedazos discretos de datos, serial, puesto que ejecuta una serie de instrucciones una tras otra, y en gran medida inflexible, ya que sigue un ciclo fijo de operaciones, en cambio la inteligencia artificial conexionista es análo$g a$, con valores continuos, paralela, puesto que procesa varias instrucciones al mismo tiempo, y en gran medida flexible, ya que sus redes tienen una gran flexibilidad de ejecución. La inteligencia artificial conexionista diseña sus programas como redes de computación paralela que imitan (aunque muy de lejos) las redes de neuronas de nuestros cerebros. Pues bien, Paul Churchland (y su esposa) cree que la inteligencia artificial conexionista promete crear máquinas con auténtico pensamiento. En consecuencia, Churchland debe admitir procesos mentales mecánicos, independientes de los procesos neurológicos, lo cual entra en contradicción con la defensa de la identificación estricta entre procesos mentales y procesos neurológicos, que sería la razón definitiva para eliminar la psicología en favor de la neurociencia.

\section{EL MATERIALISMO MENTAL DE SOBREVENIENCIA}

El filósofo coreano-americano Jaegwon Kim ha propuesto lo que denomina fisicalismo de sobreveniencia (supervenience) para aclarar las relaciones entre lo mental y lo físico, entendiendo por «físico» tanto la dimensión biológica como

23 Para mayores detalles, véase mi trabajo «Historia y filosofia de la inteligencia artificial» (2004). 
la física y química ${ }^{24}$. Sin embargo, tal como veremos, se trata de un nuevo tipo de materialismo mental, es decir, de cierta reducción de los procesos mentales a los procesos cerebrales, aunque una reducción débil, ya que mantiene cierta dualidad entre las propiedades mentales y las propiedades físicas, lo cual hace posible (podemos añadir) una psicología con cierta independencia de la neurociencia.

Los trabajos de Kim son un ejemplo de filosofía analítica, con numerosas distinciones y precisiones, razón por la cual me centraré en la exposición que presenta en su libro de texto Philosophy of Mind (1996), donde resume sus puntos de vista.

Para empezar, Kim defiende lo que denomina un fisicalismo mínimo (podríamos traducir por «materialismo débil» $»^{25}$ ). Este fisicalismo comprende tres principios $^{26}$. En primer lugar, el principio de sobreveniencia mente-cuerpo, que establece que lo mental sobreviene (supervenes) a lo físico, en el sentido de que dos cosas cualesquiera (objetos, eventos, organismos, personas, etc.) exactamente semejantes en todas las propiedades físicas no pueden diferir respecto de las propiedades mentales, de tal manera que la indiscernibilidad física entraña la indiscernibilidad psicológica. En segundo lugar, tenemos el principio anti-cartesiano, que dice que no puede haber seres puramente mentales, es decir, nada puede tener una propiedad mental sin tener una propiedad física y por ende sin ser una cosa física. Y en tercer lugar, el principio de dependencia mente-cuerpo, según el cual las propiedades mentales que tiene una cosa dada depende de, y está determinado por, las propiedades físicas que tiene, es decir, el carácter psicológico de una cosa está enteramente determinado por su carácter físico. Kim añade que esta tesis de dependencia es importante porque es una afirmación explícita de la primacía o prioridad ontológica de lo físico respecto de lo mental.

En cuanto al fisicalismo de sobreveniencia, Jaegwon Kim comienza por explicar la propia noción de sobreveniencia ${ }^{27}$. Supongamos que queremos realizar una escultura de mármol, dotada de ciertas cualidades estéticas (belleza, carácter expresivo), trabajando físicamente un trozo de mármol. Una vez terminado el trabajo físico ya no queda ningún trabajo estético por hacer, y esto es así porque las propiedades físicas del objeto determinan enteramente sus propiedades estéticas. Las propiedades estéticas de un objeto o de una situación

24 Jaegwon Kim, Philosophy of Mind (1996), p. 216.

25 En su trabajo «The myth of nonreductive materialism» (1989), Kim emplea indistintamente materialismo y fisicalismo, tal como puede verse comparando las páginas 279 y 284 .

26 Philosophy of Mind (1996), pp. 9-13.

27 O. c., pp. 222-223. 
sobrevienen a sus propiedades físicas. Podemos ahora formular el fisicalismo de sobreveniencia en la siguiente tesis ${ }^{28}$ : (FS1) Las propiedades mentales sobrevienen a las propiedades físicas en cuanto que para cualquier propiedad mental $M$, si algo tiene $M$ tiene una propiedad física $F$ tal que necesariamente si algo tiene $F$ tiene $M$. Kim añade que la expresión modal «necesariamente» puede entenderse como necesidad metafísica, y entonces la propiedad de base (o subveniente) «hace necesaria» la propiedad mental sobreveniente, o puede entenderse como necesidad nomológica, y entonces la propiedad de base será empíricamente suficiente para la propiedad mental. Esta tesis es, a su vez, equivalente a la siguiente: (FS2) Las propiedades mentales sobrevienen a las propiedades físicas en cuanto que si cualesquiera $x$ (en cualquier mundo posible) e $y$ (en cualquier mundo posible) tienen las mismas propiedades físicas (en sus respectivos mundos), entonces $x$ e $y$ tienen las mismas propiedades mentales (en esos mundos).

El materialismo de sobreveniencia, podemos comentar, distingue entre propiedades mentales y propiedades físicas, con lo cual queda abierta la posibilidad de la psicología como disciplina independiente de la neurociencia. Pero al mismo tiempo establece una dependencia de las propiedades mentales respecto de las propiedades físicas, con lo que tal independencia posible queda condicionada. En realidad, la dualidad posible entre propiedades mentales y propiedades físicas parece ser de naturaleza epistemológica, y no ontológica. Es decir, ontológicamente tendríamos que sólo hay propiedades físicas, pero desde el punto de vista del conocimiento podríamos distinguir entre propiedades físicas (neuronales), objeto de la neurociencia, y propiedades mentales, objeto de la psicología.

Jaegwon Kim vacila entre un fisicalismo no reductivo (que combina monismo fisicalista ontológico y dualismo de propiedade ${ }^{29}$ ) y un fisicalismo reductivo. En una nota en su trabajo «Postscripts on supervenience» (1993), dice que la no-reducibilidad es un asunto controvertido y es mejor dejarla fuera del concepto de sobreveniencia ${ }^{30}$. A su vez, en Philosophy of Mind (1996) declara que «aunque la sobreveniencia quizás no implica la reducibilidad, no es necesario tomarla tampoco implicando irreducibilidad, es decir, la sobreveniencia basta para los propósitos del fisicalista no-reductivo si es consistente tanto con la reducibilidad como con la irreducibilidad ${ }^{31}$. Con todo, para facilitar un fisicalismo no-reductivo formula la tesis de la sobreveniencia global $^{32}$ : (SG)

28 O. c., pp. $223-226$.

29 O. c., p. 228.

30 Supervenience and Mind (1993), nota 5 de p. 165.

31 Philosophy of Mind (1996), p. 223.

32 O.c., p. 225. 
Las propiedades mentales sobrevienen globalmente a las propiedades físicas en cuanto que mundos que son físicamente indiscernibles son también psicológicamente indiscernibles; de hecho mundos físicamente indiscernibles son uno y el mismo mundo. Esta tesis es de sobreveniencia global porque se aplica a mundos enteros y no a individuos dentro de ellos (como las anteriores FS1 y FS2). Y facilita el fisicalismo no-reductivo porque, al hablar globalmente del carácter físico y mental de mundos enteros, no parece requerir correlaciones directas de propiedades mentales específicas con propiedades físicas especíicas.

En suma, en Kim encontramos un materialismo mental que deja abierta la posibilidad de una psicología independiente de la neurociencia, ya que, además de mantener un dualismo de propiedades físicas y mentales, la no-reducción de la psicología a la neurociencia también queda abierta en virtud de la sobreveniencia global.

Sin embargo, a mi entender, la autonomía de la psicología (que no está reñida con su apoyo en la neurociencia) no queda garantizada debidamente tampoco por este materialismo mental débil. La única garantía sería dar un paso más, desde la sobreveniencia, y llegar al emergentismo. Kim contempla esta posibilidad, pero entiende que el emergentismo es cosa del pasado, habiendo florecido en la primera mitad del siglo $\mathrm{XX}^{33}$. De hecho, en su libro de texto, no se ocupa del emergentismo actual más conocido, el de John Searle, quien le corresponde declarando, en su libro The Rediscovery of the Mind (1992), que la noción de sobreveniencia ya no desempeña ningún papel en filosofía ${ }^{34}$. Para Jaegwon Kim, el emergentismo consta de tres tesis ${ }^{35}:$ 1) fisicalismo ontológico, según el cual todo lo que existe en el mundo espacio-temporal son las partículas básicas reconocidas por la física y sus agregados, 2) emergencia de propiedades, que sostiene que cuando agregados de partículas materiales alcanzan un nivel apropiado de complejidad estructural, propiedades genuinamente nuevas emergen para caracterizar estos sistemas estructurados, y 3 ) irreducibilidad de lo emergente, que dice que las propiedades emergentes son irreducibles a, e impredecibles desde, los fenómenos de nivel inferior a partir de los cuales emergen. Debemos observar, por nuestra parte, que la primera tesis defiende un materialismo ontológico, pero las otras dos tesis son contrarias al materialismo mental (o materialismo en filosofía de la mente), ya que conciben las propiedades mentales como nuevas e irreducibles respecto de las

33 O. c., p. 226. Sin embargo, en un libro algo posterior, Mind in a Physical World (1998), Kim habla del retorno del emergentismo, no sólo en la literatura filosófica seria sino también en escritos de psicología o ciencia cognitiva (pp. 8-9).

34 The Rediscovery of the Mind (1992), p. 126.

35 Philosophy of Mind (1996), pp. 226-229.

Contrastes vol. IX (2004) 
propiedades cerebrales, permitiendo así la autonomía de la psicología respecto de la neurociencia.

\section{CONCLUSIONES}

Después de este recorrido por las distintas posiciones materialistas en filosofía de la mente, todas ellas coincidentes en reducir en mayor o menor medida la psicología a la neurociencia, podemos establecer algunas conclusiones.

Tal como hemos visto, el materialismo mental clásico o teoría de la identidad mente-cerebro no elimina la psicología, pero posibilita su reducción, en última instancia, a la neurociencia. Aunque sea posible un primer análisis de los fenómenos psicológicos prescindiendo de sus equivalentes neurológicos, la definitiva y auténtica explicación de los mismos es en términos de conceptos y entidades neuronales. En suma, para el materialismo mental clásico, cabe decir, la psicología subsiste como una "vanguardia» del ejército científico que debe dejar paso a las «tropas victoriosas» de la neurociencia.

A su vez, el materialismo mental eliminativo es más radical, ya que propone, en cuanto la neurociencia se encuentre adecuadamente desarrollada, prescindir sin más de cualquier consideración psicológica y atenernos a la neurociencia para explicar los denominados procesos mentales.

Finalmente, el materialismo de sobreveniencia constituye una posición de compromiso, que permite la subsistencia de la psicología al lado de la neurociencia, en cuanto que podemos distinguir entre propiedades mentales y propiedades físicas, de tal modo que también la psicología puede generar leyes explicativas de la conducta humana ${ }^{36}$. Pero, en cuanto que el dualismo entre propiedades mentales y propiedades físicas va acompañado de un monismo ontológico materialista (implicado por el materialismo mental), la psicología puede quedar reducida a la neurociencia.

Puestas así las cosas, parece que ha llegado el momento de que enseñe mis propias cartas. La posición que defiendo constituye una combinación de funcionalismo y de emergentismo ${ }^{37}$, que denominaré funcionalemergentismo. En primer lugar, sostengo (como he indicado antes) que no sólo existen mentes animales (incluidas las humanas) sino también mentes mecánicas, es decir, que no sólo se dan procesos mentales con sistemas de neuronas como soporte físico, sino también procesos mentales con circuitos integrados electrónicos como soporte físico. En consecuencia, debe aceptarse la posición funcionalista. El funcionalismo es la doctrina filosófica de que los procesos mentales no son

36 Véase Jaegwon Kim, Mind in a Physical World (1998), p. 31.

37 Véase mi La nueva filosofia de la mente (2002), p. 142. 
conducta externa públicamente observable (frente al conductismo), sino que son funciones mediadoras entre entradas sensoriales y salidas motoras, que se producen en el interior de un sistema cognitivo y son causa de su conducta externa; como «funciones mediadoras» se encuentran mediando entre entradas y salidas, pero también están en función de las entradas y de la organización funcional del sistema; finalmente los procesos mentales pueden ser caracterizados según la función (papel causal) que desempeñan, con independencia del soporte físico del sistema, de tal manera que si se trata, por ejemplo, de demostrar un teorema, lo relevante es el conjunto de operaciones y datos de que disponemos, siendo en principio indiferente que la demostración sea ejecutada en un computador o en un cerebro humano. Esta posición funcionalista, que, para evitar malentendidos, denomino funcionalismo cognitivo, está unida de manera natural a la hipótesis empírica del sistema de símbolos físico (o del sistema de procesamiento de información, pues son lo mismo) que unifica las ciencias cognitivas $^{38}$.

En segundo lugar, la relación entre el soporte físico de un sistema, animal o mecánico, y sus procesos mentales está bastante bien definida por el emergentismo. Dicho en pocas palabras, los procesos mentales en humanos y en animales son propiedades funcionales emergentes de procesos neuronales, mientras que los procesos mentales en ordenadores (adecuadamente programados) son propiedades funcionales emergentes de los diversos dispositivos de proceso electrónico.

Carl Gustav Hempel (1905-1997) y Paul Oppenheim (1885-1977), en un trabajo profusamente citado, «Studies in the Logic of Explanation» (1948), definen de manera adecuada y defienden la emergencia. Para ellos, la ocurrencia de una característica $W$ en un objeto $w$ es emergente respecto de una teoría $T$, una relación de parte $P t$ y una clase $G$ de atributos, siempre que esa ocurrencia no pueda deducirse mediante $T$ a partir de una caracterización de las partes $P t$ de $w$ respecto de todos los atributos de G. Para Hempel y Oppenheim, hay un sentido no-trivial en que los fenómenos de la vida y los fenómenos de la mente son emergentes, consistente en el enunciado de que en función de teorías microestructurales no se dispone de explicación alguna para vastas clases de fenómenos estudiados en biología y en psicología ${ }^{39}$.

Más recientemente el célebre neurocientífico americano Roger Sperry (1913-1994), oponiéndose al dualismo de Popper y de Eccles, ha defendido la

38 Para una discusión detallada de este punto y una denuncia de las confusiones introducidas por Ned Block en la noción de funcionalismo, véase mi trabajo «Base empírica y teoría funcionalista en las ciencias cognitivas» (2001).

39 Carl G. Hempel, La explicación científica (1979), pp. 264-265. 
emergencia de las propiedades mentales respecto de la neurofisiología, en su artículo titulado «Mind-Brain Interaction: Mentalism, yes; Dualism, no» (1980). Para Sperry, los fenómenos conscientes son propiedades funcionales emergentes del procesamiento cerebral que ejercen un papel controlador activo como determinantes causales al conformar los patrones de flujo de la excitación cerebral. Una vez generados a partir de eventos neuronales, los patrones y programas mentales de orden superior tienen sus cualidades subjetivas propias, y progresan, operan e interactúan mediante sus propias leyes y principios causales que son diferentes de, y no pueden reducirse a, los de la neurofisiología. $Y$ añade Sperry que las entidades mentales transcienden lo fisiológico al igual que lo fisiológico transciende lo molecular, y lo molecular a lo atómico y subatómico ${ }^{40}$.

La autonomía de la psicología respecto de la neurociencia (que no excluye, insisto, su necesaria colaboración) también es señalada por los neurocientíficos Eric Kandel (Premio Nóbel de Medicina 2000) e Irving Kupfermann en su trabajo «De las neuronas a la cognición» (1997), quienes afirman que para enfrentarse a las bases biológicas de la cognición es necesario ir más allá de las neuronas individuales y considerar cómo se procesa la información en redes neuronales, y esto requiere no sólo los métodos y aproximaciones de la neurociencia celular y de sistemas, sino también los métodos y conocimientos de la psicología cognitiva ${ }^{4 !}$.

En general, creo que puede distinguirse claramente entre base neurológica y función psicológica, con lo que también puede distinguirse claramente entre psicología y neurociencia, abandonando el materialismo en filosofía de la mente en cualquiera de las tres versiones consideradas (materialismo clásico, eliminativista y de sobreveniencia). Además desde un punto de vista filosófico entiendo que el concepto clave en psicología es el de tiempo, mientras que el concepto clave en neurociencia es el de espacio. Por ello la cronometría mental (velocidad de procesamiento, etapas de procesamiento, tiempos de reacción, etc.) es esencial en psicología ${ }^{42}$. En cambio, en la neurociencia los diversos progresos se producen en términos de localizaciones cada vez más precisas 0 , para decirlo con mayor exactitud, en términos de identificación de sistemas neuronales especializados asociados a un determinado patrón de actividad neuronal y a un espacio flexible.

40 Roger Sperry, «Mind-Brain Interaction: Mentalism, yes; Dualism, no» (1980), p. 201.

41 Kandel y Kupfermann, «De las neuronas a la cognición» (1997), p. 371.

42 A este respecto véase el importante libro del psicólogo Michael Posner titulado Chronometric Explorations of Mind (1978). 


\section{REFERENCIAS BIBLIOGRÁFICAS}

ARMSTRONG, David Malet, «The Nature of Mind», Arts, the Proceedings of the Sydney University Arts Association, 1966.

ARMSTRONG, David Malet, A Materialist Theory of the Mind. Londres: Routledge \& Kegan Paul, 1968.

ARMSTRONG, David Malet, The Mind-Body Problem. An Opinionated Introduction. Boulder (Colorado): Westview, 1999.

BORING, Edwin G., The Physical Dimensions of Consciousness. New York: The Century Co., 1933.

BUNGE, Mario, Materialismo y ciencia. Barcelona: Ariel, 1981.

CHURCHLAND, Paul, «Eliminative Materialism and the Propositional Attitudes», Journal of Philosophy, 78, 2 (1981), 67-90.

CHURCHLAND, Paul, Matter and Consciousness. A Contemporary Introduction to the Philosophy of Mind. Cambridge (MA): The MIT Press, 1984 (1988, ed. revisada).

CHURCHLAND, Paul M. y CHURCHLAND, Patricia S., «Could a Machine Think?», Scientific American, vol. 262, 1 (1990), 32-37.

FEYERABEND, Paul, «Materialism and the Mind-Body Problem», The Review of Metaphysics, XVII, 1 (1963), 49-66.

FODOR, Jerry, Psychosemantics. The Problem of Meaning in the Philosophy of Mind. Cambridge (MA): The MIT Press, 1987.

HEISENBERG, Werner, La imagen de la naturaleza en la física actual, tr. Gabriel Ferraté. Barcelona: Planeta-Agostini, 1993.

HEMPEL, Carl Gustav, y OPPENHEIM, Paul, «Studies in the Logic of Explanation», Philosophy of Science, 15 (1948), 135-175. Reimpreso en Carl G. Hempel, La explicación científica. Estudios sobre la filosofia de la ciencia, tr. Irma Ruiz Aused. Buenos Aires: Paidós, 1979, 247-294.

KANDEL, Eric, y KUPFERMANN, Irving, «De las neuronas a la cognición», en E. Kandel y otros, Neurociencia y conducta, tr. Pilar Herreros y otros. Madrid: Prentice Hall, 1997, 345-372.

KIM, Jaegwon, «The myth of nonreductive materialism», en J. Kim, Supervenience and Mind. Selected Philosophical Essays. New York: Cambridge University Press, 1993, 265-284.

KIM, Jaegwon, «Postscripts on supervenience», en J. Kim, Supervenience and Mind. Selected Philosophical Essays. New York: Cambridge University Press, 1993, 161-171.

KIM, Jaegwon, Philosophy of Mind. Boulder (Colorado): Westview Press, 1996.

KIM, Jaegwon, Mind in a Physical World. An Essay on the Mind-Body Problem and Mental Causation. Cambridge (MA): The MIT Press, 1998.

LEWIS, David, «An Argument for the Identity Theory», Journal of Philosophy, 63 (1966), 17-25.

LEWIS, David, «Psychophysical and Theoretical Identifications», Australasian Journal of Philosophy, 50 (1972), 249-258.

MARTÍNEZ-FREIRE, Pascual F., «Aproximaciones científicas al problema de la

Contrastes vol. IX (2004) 
conciencia», en Ildefonso Murillo (ed.), Fronteras de la filosofía de cara al siglo XXI. Colmenar Viejo (Madrid): Diálogo Filosófico, 2000, 181-197.

MARTINEZ-FREIRE, Pascual F., «Mente, inteligencia y espíritu», en Luis Méndez (coord.), Ética y Sociología. Estudios en memoria del profesor José Todolí. Salamanca: Editorial San Esteban, 2000, 693-705.

MARTINEZ-FREIRE, Pascual F., «Base empírica y teoría funcionalista en las ciencias cognitivas», Agora, 20-1 (2001), 87-104 (publicado en 2002).

MARTINEZ-FREIRE, Pascual F., La nueva filosofía de la mente. Barcelona: Gedisa, 1995, 2002 (reimpresión).

MARTINEZ-FREIRE, Pascual F., «Historia y filosofía de la inteligencia artificial», en J. M. Atencia y A. Diéguez (coords.), Tecnociencia y cultura a comienzos del siglo XXI. Málaga: Universidad de Málaga, 2004, 179-226.

PLACE, Ullin T., «Is Consciousness a Brain Process?», British Journal of Psychology, XLVII (1956), 44-50.

PLACE, Ullin T., «A Pilgrim's Progress. From Mystical Experience to Biological Consciouness», Journal of Consciousness Studies, 9, 3 (2002), 3452.

POSNER, Michael, Chronometric Explorations of Mind, New York: Oxford University Press, 1978.

SEARLE, John, The Rediscovery of the Mind. Cambridge (MA): The MIT Press, 1992.

SMART, John J. C., «Sensations and Brain Processes», Philosophical Review, LXVIII (1959), 141-156.

SPERRY, Roger, «Mind-Brain Interaction: Mentalism, yes; Dualism, no», Neuroscience 5 (1980), 195-206.

Pascual F. Martínez-Freire es catedrático de Lógica y Filosofía de la Ciencia, y responsable del Grupo de Investigación en Ciencias Cognitivas, de la Universidad de Málaga. Últimamente ha publicado «Historia y filosofía de la inteligencia artificial», en J. Ma. Atencia y A. Diéguez (coords.) Tecnociencia y cultura a comienzos del siglo XXI, Universidad de Málaga, 2004, y "Popper y el problema cuerpo-mente», en A. Rivadulla (ed.), Hipótesis y verdad en ciencia. Ensayos sobre la filosofía de Karl R. Popper, Universidad Complutense, 2004.

Dirección postal: Departamento de Filosofía, Facultad de Filosofía y Letras, Campus de Teatinos, E-29071 Málaga.

E-mail: freire@uma.es

http://webdeptos.uma.es/filosofia/freire.htm 